\title{
The role of aerodynamic drag in propagation of interplanetary coronal mass ejections
}

\author{
B. Vršnak ${ }^{1}$, T. Žic ${ }^{1}$, T. V. Falkenberg ${ }^{2}$, C. Möstl ${ }^{3,4}$, S. Vennerstrom ${ }^{2}$, and D. Vrbanec ${ }^{1}$ \\ 1 Hvar Observatory, Faculty of Geodesy, University of Zagreb, Kačićeva 26, 10000 Zagreb, Croatia \\ e-mail: bvrsnak@geof.hr \\ 2 National Space Institute, Technical University of Denmark, Juliane Maries vej 30, 2100 København, Denmark \\ 3 Space Research Institute, Austrian Academy of Sciences, Schmiedlstrasse 6, 8042 Graz, Austria \\ ${ }^{4}$ Institute for Physics, University of Graz, Universitätsplatz 5, 8010 Graz, Austria \\ Received 15 October 2009 / Accepted 17 December 2009
}

\begin{abstract}
Context. The propagation of interplanetary coronal mass ejections (ICMEs) and the forecast of their arrival on Earth is one of the central issues of space weather studies.

Aims. We investigate to which degree various ICME parameters (mass, size, take-off speed) and the ambient solar-wind parameters (density and velocity) affect the ICME Sun-Earth transit time.

Methods. We study solutions of a drag-based equation of motion by systematically varying the input parameters. The analysis is focused on ICME transit times and 1 AU velocities.

Results. The model results reveal that wide ICMEs of low masses adjust to the solar-wind speed already close to the sun, so the transit time is determined primarily by the solar-wind speed. The shortest transit times and accordingly the highest 1 AU velocities are related to narrow and massive ICMEs (i.e. high-density eruptions) propagating in high-speed solar wind streams. We apply the model to the Sun-Earth event associated with the CME of 25 July 2004 and compare the results with the outcome of the numerical MHD modeling.
\end{abstract}

Key words. Sun: coronal mass ejections (CMEs) - solar-terrestrial relations - solar wind - magnetohydrodynamics (MHD) Sun: corona

\section{Introduction}

Interplanetary coronal mass ejections (ICMEs) are drivers of major geomagnetic storms (cf. Gosling et al. 1990; Zhang et al. 2003; Koskinen \& Huttunen 2006, and references therein), so predicting their arrivals on Earth is one of the central issues of space weather. For this purpose, various forecasting methods were developed based either on the empirical/statistical relationships (e.g. Gopalswamy et al. 2000; Michałek et al. 2004; Vršnak \& Žic 2007), analytical propagation models (e.g. Smart \& Shea 1985; Vršnak \& Gopalswamy 2002; Cantó et al. 2005; Vršnak \& Žic 2007; Borgazzi et al. 2009; Lara \& Borgazzi 2009), or numerical MHD simulations (e.g. González-Esparza et al. 2003; Manchester et al. 2004; Odstrcil et al. 2004; Smith et al. 2009; Taktakishvili et al. 2009). Performances of these forecasting procedures were analyzed in a number of validation studies (e.g. Cho et al. 2003; Dryer et al. 2004; Oler 2004; McKenna-Lawlor et al. 2006; Feng et al. 2009; Smith et al. 2009; Taktakishvili et al. 2009), showing that the current prediction accuracy ranges around $\pm 10 \mathrm{~h}$ and that sometimes errors can be even larger than one day.

There are several lines of evidence indicating that the dynamics of the ICMEs is dominated by the "aerodynamic" drag (e.g. Gopalswamy et al. 2000, 2001; Vršnak \& Gopalswamy 2002; Yashiro et al. 2004; Tappin 2006; Manoharan 2006; Vršnak \& Žic 2007; Vršnak et al. 2008). Taking into account the physical properties of the drag (e.g. Cargill et al. 1996; Vršnak 2001a; Cargill 2004; Vršnak et al. 2004), it can be presumed that the ICME kinematics depend on speed, mass, and size as well as on the ambient solar-wind density and velocity. Thus the transit time depends intrinsically on a number of parameters, which causes uncertainties in forecasting the ICME arrival. In this paper we analyze to which degree various input parameters affect the outcome of a simple drag-based equation of motion, i.e., we try to find the most relevant quantities for the ICMEarrival forecast. In Sect. 2 we describe the model, the results are presented in Sect. 3, and in Sect. 4 we apply the model to a specific event, to compare the results with the outcome of the numerical MHD simulation.

\section{The model}

Generally, the CME dynamics are governed by the Lorentz force, the gravity, and the aerodynamic drag (e.g. Chen 1989; Cargill 2004), i.e., the net acceleration can be written as

$a=a_{L}-g+a_{\mathrm{d}}$

where $a_{L}, g$, and $a_{\mathrm{d}}$ represent the accelerations due to the mentioned three forces, respectively.

Furthermore, we assume that beyond the heliocentric distance of $r=20 r_{\odot}$ the contribution of the term $a_{L}-g$ becomes negligible (Vršnak 2001b; Vršnak \& Gopalswamy 2002; Vršnak et al. 2004), implying $a \approx a_{\mathrm{d}}$. The drag acceleration can be expressed in the form (see e.g. Cargill et al. 1996; Cargill 2004; Vršnak et al. 2004)

$a_{\mathrm{d}}=-\gamma(v-w)|v-w|$, 
where $v$ is the CME velocity and $w$ represents the ambient solar wind speed. According to Eq. (2), CMEs decelerate if $v>w$, or accelerate if $v<w$. The parameter $\gamma$ is usually expressed in the form

$\gamma=c_{\mathrm{d}} \frac{A \rho_{w}}{m}$,

where $c_{\mathrm{d}}$ represents the dimensionless drag coefficient, $A$ is the cross section area of the CME perpendicular to the direction of the propagation, $\rho_{w}$ is the ambient solar wind density, and $m$ is the CME mass. The area $A$ depends on the CME shape; below we will apply the CME cone model (Fisher \& Munro 1984)

$A=\pi\left(\frac{\phi}{2} r\right)^{2}$

where $\phi / 2$ represents the cone half-angle expressed in radians, i.e., $\phi$ is the CME-cone angular width (for details of the cone model see Zhao et al. 2002; Michałek et al. 2003; Xie et al. 2004; Schwenn et al. 2005; Xue et al. 2005). Below we assume that the width $\phi$ and the CME mass $m$ do not change significantly beyond $r=20 r_{\odot}$.

For the solar wind density we employ the empirical model derived by Leblanc et al. (1998)

$n_{w}(R)=\frac{8.0 \times 10^{7}}{R^{6}}+\frac{4.1 \times 10^{6}}{R^{4}}+\frac{3.3 \times 10^{5}}{R^{2}}$,

where the number density $n$ is expressed in $\mathrm{cm}^{-3}$ and the heliocentric distance is given in units of the solar radius, $R=r / r_{\odot}$. The density is normalized to $n=7 \mathrm{~cm}^{-3}$ at $1 \mathrm{AU}$; at $R=20$ this corresponds to $n=850 \mathrm{~cm}^{-3}$.

Given the function $n(R)$, the solar wind speed is defined by the equation of continuity

$w(R)=w_{1} \frac{n_{1} R_{1}^{2}}{n(R) R^{2}}$,

where the subscript " 1 " represents the values at 1 AU. Here, we assume that the solar wind speed $w$ and the solar wind density $n$ depend only on the heliocentric distance $R$, i.e. that the flow is purely radial.

Since $a=r_{\odot} \ddot{R}$ and $v=r_{\odot} \dot{R}$, Eq. (2) substituted by Eqs. (3)-(6) represents a differential equation which can be easily solved numerically. Thus, the interplanetary dynamics of our model-CME is determined by the parameters $m, \phi, n_{1}$, and $w_{1}$, as well as by the ICME take-off speed $v_{0}$, which defines the initial condition: we take that at $t=0$ the CME is located at $R=20$, with the velocity $v_{0}$.

Note that at distances beyond $R=20$ the first term on righthand-side of Eq. (5) can be neglected. Furthermore, at such distances the solar-wind speed is already very close to its asymptotic value, since the main contribution in Eq. (5) comes from the third term, $n \propto R^{-2}$, i.e., $w \approx$ const.

\section{Results}

In the next subsection we illustrate how the CME kinematics depend on the parameters $v_{0}, m, \phi, n_{1}$, and $w_{1}$. In Sect. 3.2 we focus on the $1 \mathrm{AU}$ transit-time and the $1 \mathrm{AU}$ speed. In all examples we apply $c_{\mathrm{d}}=1$ (for a discussion see Cargill 2004). Note that the parameters $m, \phi, n_{1}$, and $c_{\mathrm{d}}$ appear in Eq. (2) in the form $k=c_{\mathrm{d}} \phi^{2} n_{1} / m$. Thus any combination of these parameters that gives the same value of the parameter $k$ results in the same solution, which means basically that we have to combine only three parameters, $k, v_{0}$, and $w_{1}$. Nevertheless, we will present our results as a function of $m, \phi$, and $n_{1}$ to stay closer to the specific observable quantities.

\subsection{Kinematics}

In Figs. 1 $\mathrm{a}$ and $\mathrm{b}$ the ICME velocity is presented as a function of time and distance, respectively. We stop the calculation at $1 \mathrm{AU}$, so the $x$-coordinate of the last point of the $v(t)$ curves in Fig. 1a defines the ICME transit time $T T$ and the $y$-coordinate represents the $1 \mathrm{AU}$ speed $v_{1}$. The take-off velocity $v_{0}$ at $R_{0}=20$ (i.e., at $t=0$ ) is taken to be $v_{0}=200$ and $1000 \mathrm{~km} \mathrm{~s}^{-1}$. The 1 AU solar-wind speed and the ICME angular width are fixed to $w_{1}=400 \mathrm{~km} \mathrm{~s}^{-1}$ and $\phi=1 \mathrm{rad}$. The black-bold line $\left(v_{0}=1000 \mathrm{~km} \mathrm{~s}^{-1}\right)$ and black-thin line $\left(v_{0}=200 \mathrm{~km} \mathrm{~s}^{-1}\right)$ are calculated for the ICME mass $m=10^{12} \mathrm{~kg}$, whereas the gray-bold line represents the outcome for $v_{0}=1000 \mathrm{~km} \mathrm{~s}^{-1}$ and $m=4 \times$ $10^{12} \mathrm{~kg}$. The latter option, $m=4 \times 10^{12} \mathrm{~kg}$ with $\phi=1 \mathrm{rad}$, also corresponds to e.g. $m=10^{12} \mathrm{~kg}$ with $\phi=0.5 \mathrm{rad}$, since $k \propto \phi^{2} / m$. The dashed line indicates $w_{1}=400 \mathrm{~km} \mathrm{~s}^{-1}$ but also approximately represents $v(t)$ for the case $v_{0}=400 \mathrm{~km} \mathrm{~s}^{-1}$, since at $R>20$ the solar-wind speed is already quite close to its asymptotic value, implying that the CME acceleration is negligible $(v(t) \approx$ const.).

In Fig. 1c we show the acceleration as a function of distance for the same set of parameters as in Figs. 1 $a$ and $b$. Note that for $v_{0}=200 \mathrm{~km} \mathrm{~s}^{-1}$ the CME accelerates, for $v_{0}=400 \mathrm{~km} \mathrm{~s}^{-1}$ it moves with $a \approx 0$, and for $v_{0}=1000 \mathrm{~km} \mathrm{~s}^{-1}$ the CME decelerates. Such a behavior is consistent with measurements based on the coronagraphic data (see, e.g. Vršnak et al. 2004). Moreover, the values at $R \approx 20$ are consistent with typical accelerations measured in this distance range.

Comparing the two kinematical curves for $v_{0}=1000 \mathrm{~km} \mathrm{~s}^{-1}$, we find that the lower-mass CME is decelerated much more effectively than the massive one. Consequently, low-mass CMEs travel most of their way to the Earth practically at the solar wind speed. Given that the same results would be obtained for equal masses but different angular width $\phi$, Fig. 1 also implicitly shows that wide CMEs are decelerated much more effectively than the narrow ones. Analogously, CMEs propagating through a higher-density solar wind (larger $n_{1}$ ) are more affected by the drag. Thus, the shortest transit times can be achieved only by fast, massive, and relatively narrow CMEs (implying also high densities), especially when propagating in high speed solar wind streams of low density. The described behavior is fully consistent with the empirical relationships reported by Vršnak \& Žic (2007) and Vršnak et al. (2008).

\subsection{AU transit times and velocities}

Calculations described in Sect. 3.1 were performed over the parameter space to evaluate the $1 \mathrm{AU}$ transit times, $T T$, and the ICME velocity at $1 \mathrm{AU}, v_{1}$, as a function of $v_{0}, m, \phi, n_{1}$, and $w_{1}$. In Figs. $2 \mathrm{a}$ and $\mathrm{b}$ we present the dependencies $T T\left(v_{0}\right)$ and $v_{1}\left(v_{0}\right)$, for different solar wind speeds $w_{1}$ and fixed values $\phi=1$ and $m=10^{12} \mathrm{~kg}$. As already mentioned, the outcome is the same for e.g. $\phi=2$ and $m=4 \times 10^{12} \mathrm{~kg}$, or $\phi=2$ and four times lower solar wind density and consequently for any combination that gives the same value of the parameter $k$. As expected, transit times become longer for lower solar wind speeds. Note also that for the considered set of parameters, the transit time $T T$ is affected more by the solar wind speed than by the CME takeoff speed $v_{0}$. This is primarily due to the fast adjustment of the CME velocity to the ambient solar wind speed in the case of high- $k$ events (wide/low mass CMEs).

To emphasize the importance of the solar wind speed for high- $k$ CMEs we show in Figs. $3 \mathrm{a}$ and $\mathrm{b}$ the dependencies $T T\left(w_{1}\right)$ and $v_{1}\left(w_{1}\right)$, calculated by applying a wide range of 

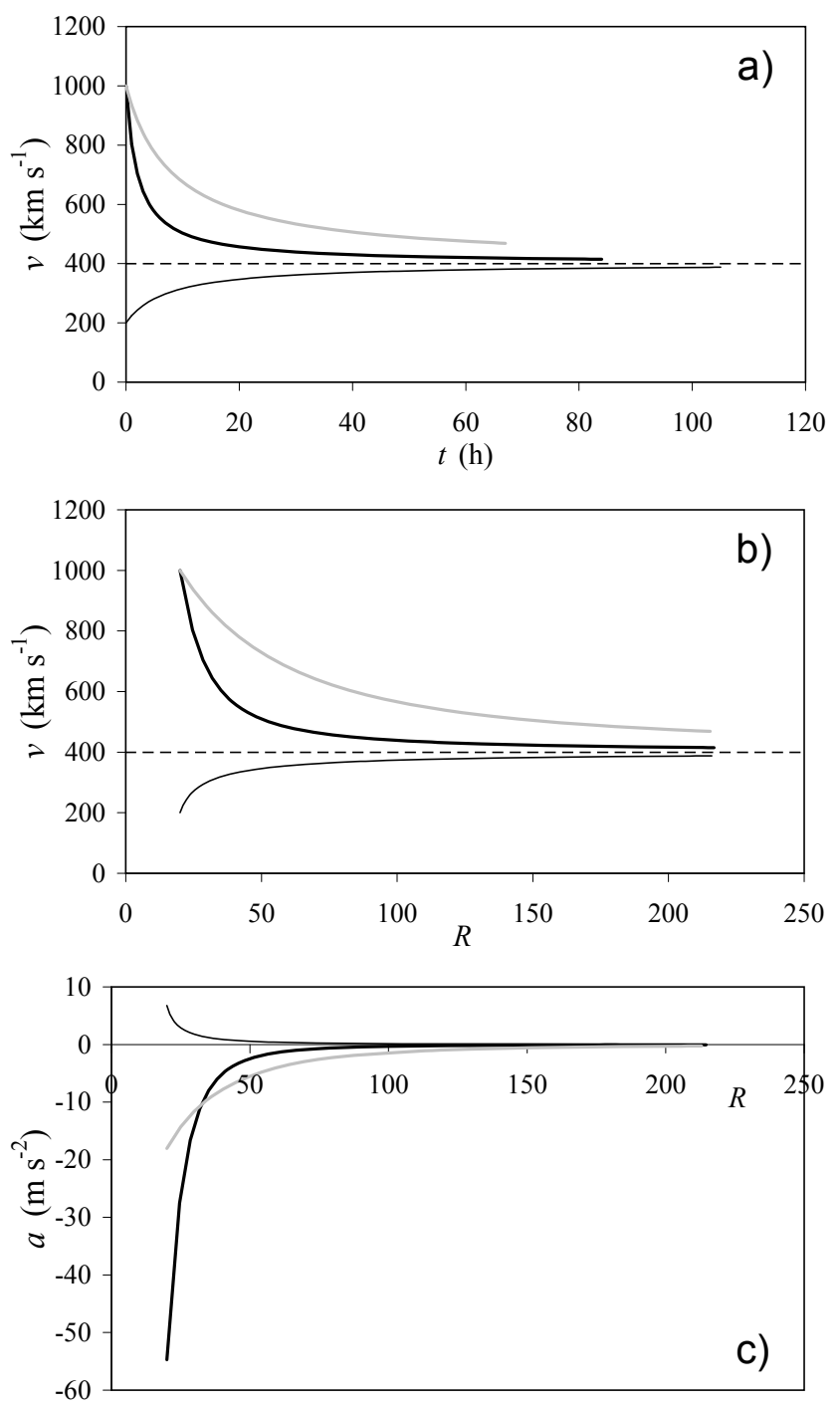

Fig. 1. CME kinematics calculated for the solar wind speed $w_{1}=$ $400 \mathrm{~km} \mathrm{~s}^{-1}$ (dashed line): a) velocity as a function of time; b) velocity as a function of distance; $\mathbf{c}$ ) acceleration as a function of distance. Thin line represents $v_{0}=200 \mathrm{~km} \mathrm{~s}^{-1}$ and $m=10^{12} \mathrm{~kg}$, black-bold line $v_{0}=1000 \mathrm{~km} \mathrm{~s}^{-1}$ and $m=10^{12} \mathrm{~kg}$, bold-gray line $v_{0}=1000 \mathrm{~km} \mathrm{~s}^{-1}$ and $m=4 \times 10^{12} \mathrm{~kg}$.

take-off speeds $v_{0}$. The presented graphs clearly illustrate that transit times and 1 AU speeds of high- $k$ CMEs depend much more on $w_{1}$ than on $v_{0}$.

The influence of the take-off speed becomes greater for CMEs of larger masses (or equivalently narrower CMEs, which propagate in low density solar wind). In Figs. $4 \mathrm{a}$ and b we show the dependencies $T T\left(v_{0}\right)$ and $v_{1}\left(v_{0}\right)$ for different values of $\phi$, and the solar-wind speed of $w_{1}=400 \mathrm{~km} \mathrm{~s}^{-1}$. The graph shows that for narrow CMEs (low- $k$ events), the transit time becomes much more dependent on the initial speed than it was in the case of broad CMEs (high- $k$ events). The same holds for the 1 AU speed.

The conclusions about "low- $k$ " versus "high- $k$ " CMEs based in Figs. 2-4 are directly quantified by graphs presented in Figs. 5a and $b$. Here we show how the transit time and 1 AU speed depend on the CME width (or equivalently the CME mass), where we have fixed the solar wind speed to $w_{1}=$ $400 \mathrm{~km} \mathrm{~s}^{-1}$ and applied various take-off speeds $v_{0}$. Figure 5 clearly shows that for wide CMEs (equivalently, low-mass CMEs) the transit time and the $1 \mathrm{AU}$ speed do not depend
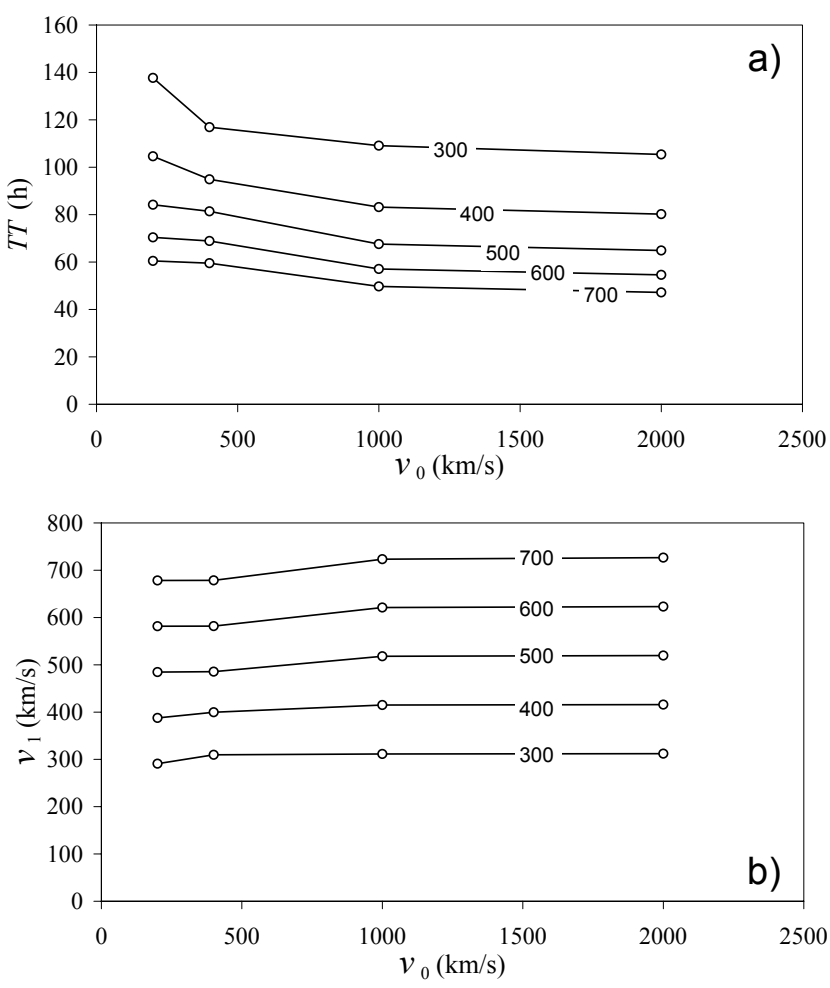

Fig. 2. a) Transit time $T T$ presented as a function of the ICME take-off speed $v_{0}$, calculated for $\phi=1 \mathrm{rad}, m=10^{12} \mathrm{~kg}$, and different solar wind speeds $w_{1}$ (written in $\mathrm{km} \mathrm{s}^{-1}$ by the curves); b) the ICME velocity at $1 \mathrm{AU}$ for the same set of input parameters.

significantly on the take-off speed $v_{0}$. The initial speed is relevant only for low- $k$ events, i.e., narrow and massive CMEs.

The strong influence of the solar wind speed on the transit time straightforwardly explains the so-called "Brueckner's 80-h rule" ( $T T \approx 80 \mathrm{~h}$ in most events; Brueckner et al. 1998). In the majority of events, the CME segments that hit Earth propagate through the slow solar wind $\left(w_{1} \approx 400 \mathrm{~km} \mathrm{~s}^{-1}\right)$. Figure 3 shows that under such circumstances a typical transit time for an "average" CME $\left(v_{0}=300-500 \mathrm{~km} \mathrm{~s}^{-1}\right.$; see e.g. Yashiro et al. 2004) ranges between 60 and $100 \mathrm{~h}$. Furthermore, our results show that the shortest transit times ( $T T \lesssim 1$ day; for a discussion see Gopalswamy et al. 2005) can be achieved only by massive CMEs of a very high initial speed $\left(v_{0}>2000 \mathrm{~km} \mathrm{~s}^{-1}\right)$. In addition, such a CME has to move through high-speed solar wind streams and the Lorentz force has to act over long distances to postpone the drag-dominated phase until the solar wind density becomes low.

Finally it should be noted that throughout this section we were using the CME mass $m$ and the angular width $\phi$ as basic parameters, primarily because they can be estimated from the observations. However, the drag parameter $\gamma$ can also be expressed in the form

$\gamma=c_{\mathrm{d}} \frac{A}{V} \frac{\rho_{w}}{\rho_{\mathrm{CME}}}$,

where $V$ and $\rho_{\mathrm{CME}}$ are the CME volume and density (for a discussion see Cargill 2004). Thus, massive/narrow CMEs, which are less affected by the drag, are in fact CMEs whose density is significantly higher than the solar wind density. 

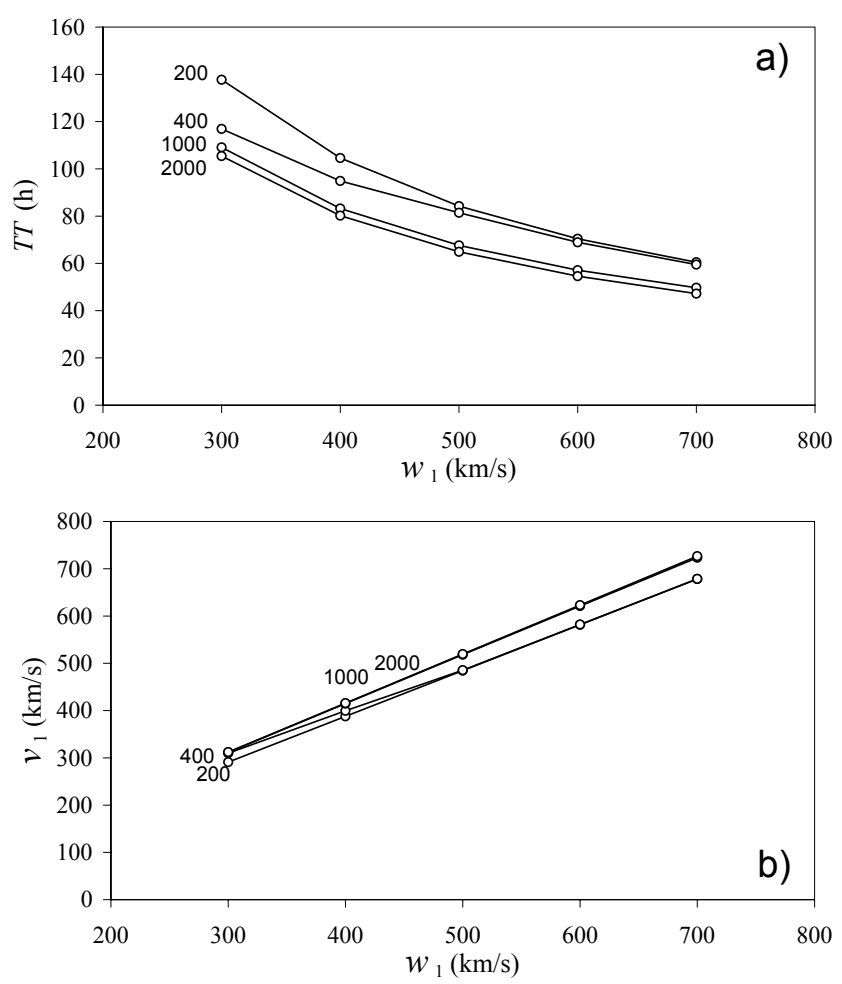

Fig. 3. a) Transit time $T T$ presented as a function of the solar wind speed $w_{1}$, calculated for $\phi=1 \mathrm{rad}, m=10^{12} \mathrm{~kg}$ and different values of the ICME take-off speed $v_{0}$ (written in $\mathrm{km} \mathrm{s}^{-1}$ by the curves); b) the ICME velocity at $1 \mathrm{AU}$ for the same set of input parameters.
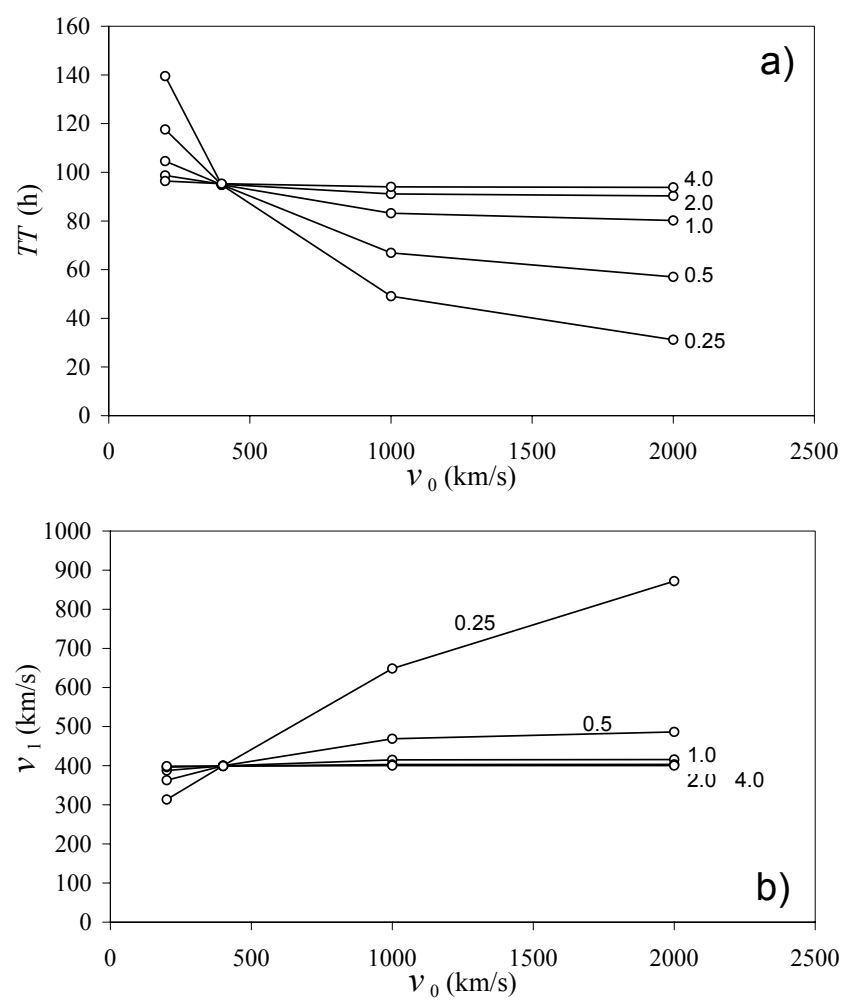

Fig. 4. a) Transit time $T T$ presented as a function of the ICME take-off speed $v_{0}$, calculated for $w_{1}=400 \mathrm{~km} \mathrm{~s}^{-1}, m=10^{12} \mathrm{~kg}$, and different values of $\phi$ (written in radians attached to the curves); b) The ICME velocity at $1 \mathrm{AU}$ for the same set of input parameters. The same result would be obtained by using fixed $\phi=1 \mathrm{rad}$ and masses from $6.25 \times$ $10^{10}$ to $1.6 \times 10^{13} \mathrm{~kg}$ (corresponding to $\phi=4-0.25 \mathrm{rad}$ respectively).
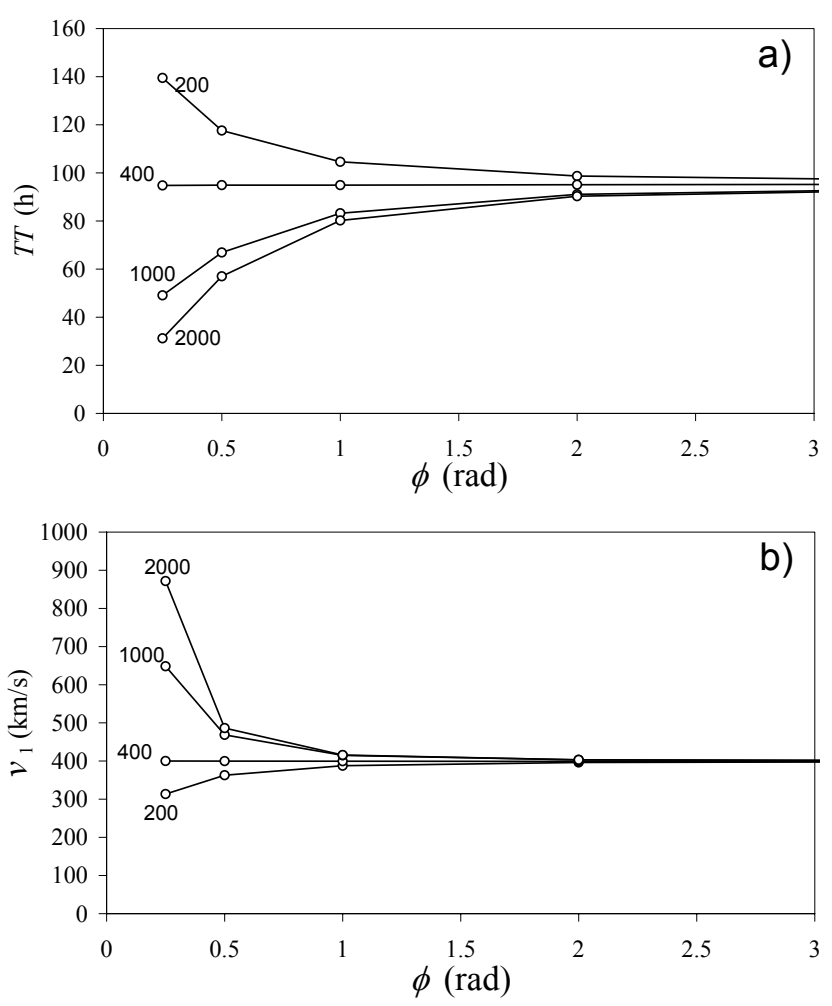

Fig. 5. a) Transit time $T T$ presented as a function of the angular size of the CME, $\phi$, calculated for $w_{1}=400 \mathrm{~km} \mathrm{~s}^{-1}, m=10^{12} \mathrm{~kg}$ and different values of the ICME take-off speed $v_{0}$ (written by the curves); b) the ICME velocity at $1 \mathrm{AU}$ for the same set of input parameters.

\section{An example and comparison with numerical results}

\subsection{Application of the model to the CME of 25 July 2004}

To demonstrate the application of the model, we utilize observations of the halo CME of 25 July 2004 (first appearance in the LASCO$^{1}$ field-of-view at 14:54 UT). This was one of the SunEarth events foreseen for a detailed interdisciplinary study in the frame of the European FP-7 project SOTERIA (Solar-Terrestrial Investigations and Archives; http://soteria-space.eu/). We have chosen this event, associated with the $D s t \approx-200 \mathrm{nT}$ geomagnetic storm on 27 July 2007, because of a relatively simple global situation, yet including the presence of the solar wind high speed stream. A brief description of the event was presented by Yousef et al. (2005a,b).

The CME was launched from the active region NOAA 10652 and was associated with a $1 \mathrm{~F} / \mathrm{M} 1$ flare located at n08w33. This long duration flare started at $\approx 14: 20 \mathrm{UT}$, after a series of impulsive compact flares occurring over a period of two hours. The $1-8 \AA$ soft X-ray flux peaked at $\approx 15: 20 \mathrm{UT}$, and during the decay phase, the Solar X-ray Imager (SXI; Pizzo et al. 2005) onboard the GOES satellite showed a huge cusped post-eruption loop-system south of NOAA 10652, revealing that the eruption occurred in between NOAA 10652 and NOAA 10653.

In the LASCO field of view the CME had an approximately constant velocity of $v=1330 \mathrm{~km} \mathrm{~s}^{-1}$, reaching the heliocentric distance of $R=20$ at 17:28 UT. Below we express the time in the "day-of-year" form, $D O Y$, so the CME was at $R=20$ at $D O Y=207.73$.

1 The Large Angle Spectroscopic Coronagraph (LASCO; Brueckner et al. 1995) 
The 1 AU data measured by the Advanced Composition Explorer (ACE) and Wind spacecrafts reveal the arrival of the ICME-associated shock at $D O Y=208.94$, which is 1.2 days $(28.8 \mathrm{~h})$ after the CME take-off. According to the measured magnetic field pattern, the leading edge of the ICME arrived at $D O Y \approx 209.09 \pm 0.02$. Thus the ICME transit time from $R=20$ was $T T=1.36$ days or $32.6 \mathrm{~h}$. The 1 AU ICME speed, defined as the peak velocity within the magnetic cloud, was $v_{1}=1000 \pm 20 \mathrm{~km} \mathrm{~s}^{-1}$.

To explicitly solve the equation of motion we need to know the CME mass and angular width as well as the solar wind speed and density at $1 \mathrm{AU}$. The CME brightness in calibrated base-difference LASCO images is dependent on the number of Thompson-scattering electrons, which provides an estimate of the CME mass (for details see e.g. Vourlidas et al. 2000). The mass reported in the LASCO online catalog (http://cdaw. gsfc. nasa.gov/CME_list/; Yashiro et al. 2004) for our CME equals $m=1.1 \times 10^{13} \mathrm{~kg}$.

The angular width of a halo CME can be estimated from its shape by assuming the cone model geometry. Applying the method proposed by Xie et al. (2004) we get $\phi=150^{\circ}=2.6 \mathrm{rad}$. On the other hand, inspecting the CME-associated coronal dimming, the angular width can be estimated as $\phi \approx 1.5 \mathrm{rad}$. The minimum angular width can be also estimated from the source region location and the demand that it results in the halo $\mathrm{CME}$, or more-or-less equivalently, that it encounters the Earth. Given the associated-flare location, from this demand we get $\phi \gtrsim 66^{\circ}$.

Inspecting the in situ data we find that the solar wind speed ahead of ICME $(D O Y=108-109)$ was characterized by a high speed, $w_{1}=600-650 \mathrm{~km} \mathrm{~s}^{-1}$, and relatively low density, $n_{1}=2-2.5 \mathrm{~cm}^{-3}$. However, we find no Alfvénic fluctuations, implying that this was probably not a high-speed stream originating from an equatorial coronal hole, but more likely a consequence of a weak ICME. Indeed, we find a pattern indicating a rotation of the magnetic field vector, which suggests a small/weak magnetic cloud. On the other hand we find a typical high speed stream behind our ICME (after $D O Y=110$ ) characterized by the density $n_{1}=0.5-1 \mathrm{~cm}^{-3}$ and the flow speed $w_{1}=$ $650-700 \mathrm{~km} \mathrm{~s}^{-1}$. The stream has its origin in the equatorial coronal hole located eastward of the CME source site, passing over the central meridian on 26 July (for spatial/temporal relationship between coronal holes and high speed streams see e.g. Vršnak et al. 2007, and references therein). Thus, considering the situation ahead and behind the ICME, we use $w_{1}=650 \mathrm{~km} \mathrm{~s}^{-1}$.

Given the presented observations/measurements we take for the model input

$-v_{0}=1330 \mathrm{~km} \mathrm{~s}^{-1}$ at $R_{0}=20$;

- $\phi=1.1-2.6 \mathrm{rad}$

- $m=1.1 \times 10^{13} \mathrm{~kg}$;

$-w_{1}=650 \mathrm{~km} \mathrm{~s}^{-1}$

- $n_{1}=0.5-2.5 \mathrm{~cm}^{-3}$, corresponding to 14-3 times lower density than in the model of Leblanc et al. (1998).

A summary of calculated transit times $T T_{\mathrm{C}}$ and $1 \mathrm{AU}$ velocities $v_{1 \mathrm{C}}$ is shown in Table 1 , where we present the outcome for various combinations of maximum, mean, and minimum values of $\phi$ and $n_{1}$ (shown in the first two columns). In Cols. 3-5 we present the calculated transit times $T T_{\mathrm{C}}$, the difference between the observed and calculated values $\Delta T T \equiv T T-T T_{\mathrm{C}}$, and the relative difference $\Delta T T / T T$, respectively. Columns 6-8 show the calculated $1 \mathrm{AU}$ velocities $v_{1 \mathrm{C}}$, the difference between the observed and calculated speeds $\Delta v_{1} \equiv v_{1}-v_{1 \mathrm{C}}$, and the relative difference $\Delta v_{1} / v_{1}$, respectively. Negative values mean that the calculated arrival is delayed with respect to the observed
Table 1. Summary of calculated transit times $T T_{\mathrm{C}}$ and $1 \mathrm{AU}$ velocities $v_{1 \mathrm{C}}$.

\begin{tabular}{lrccrrrr}
\hline \hline $\begin{array}{l}l \\
\mathrm{rad}\end{array}$ & $\begin{array}{r}n_{1} \\
\mathrm{~cm}^{-3}\end{array}$ & $\begin{array}{c}T T_{\mathrm{C}} \\
\mathrm{h}\end{array}$ & $\begin{array}{c}\Delta T T \\
\mathrm{~h}\end{array}$ & $\begin{array}{r}\frac{\Delta T T}{T T} \\
\%\end{array}$ & $\begin{array}{r}v_{1 \mathrm{C}} \\
\mathrm{km} \mathrm{s}^{-1}\end{array}$ & $\begin{array}{r}\Delta v_{1} \\
\mathrm{~km} \mathrm{~s}^{-1}\end{array}$ & $\begin{array}{r}\frac{\Delta v_{1}}{v_{1}} \\
\%\end{array}$ \\
\hline 2.6 & 2.5 & 42.4 & -9.8 & -30 & 765 & 235 & 23 \\
2.6 & 1.5 & 34.2 & -1.6 & -5 & 973 & 27 & 3 \\
1.8 & 2.5 & 37.2 & -4.6 & -14 & 871 & 129 & 13 \\
2.6 & 0.5 & 32.7 & -0.1 & 0 & 1041 & -41 & -4 \\
1.8 & 1.5 & 38.5 & -5.9 & -18 & 838 & 162 & 16 \\
1.1 & 2.5 & 32.4 & 0.2 & 0 & 1056 & -56 & -6 \\
1.8 & 0.5 & 30.6 & 2.0 & 6 & 1161 & -161 & -16 \\
1.1 & 1.5 & 30.9 & 1.7 & 5 & 1145 & -145 & -14 \\
1.1 & 0.5 & 29.3 & 3.3 & 10 & 1258 & -258 & -26 \\
\hline
\end{tabular}

one, or that the calculated $1 \mathrm{AU}$ velocity is higher than the observed one.

Inspecting Table 1, one finds that the values of $\phi$ and $n_{1} \mathrm{em}-$ ployed in the model runs cover a broad range of values of the parameter $k \propto \phi^{2} n_{1}$, changing almost by a factor of 30 . Such a wide range of $k$ should compensate for uncertainties in determining the CME mass and the true (deprojected) CME speed, as well as possible deviations from $c_{\mathrm{d}}=1$.

From Table 1 we find that the time window of calculated transit times is only $13 \mathrm{~h}$ : we get the limits $T T=29-42 \mathrm{~h}$, corresponding to $v_{1}=1270-770 \mathrm{~km} \mathrm{~s}^{-1}$, respectively. The best match $(\Delta T T=0 \mathrm{~h})$ is obtained for: a) $\phi=2.6 \mathrm{rad}$, determined from the observations using the CME cone-model and the measured high-speed stream density of $n_{1}=0.5 \mathrm{~cm}^{-3}$; b) the lowerlimit width, $\phi=1.1 \mathrm{rad}$, and the upper-limit solar-wind density $n_{1}=2.5 \mathrm{~cm}^{-3}$. The largest deviation, $\Delta T T=-9.8 \mathrm{~h}$, is found for the extreme combination $\phi=2.6 \mathrm{rad}$ and $n_{1}=2.5 \mathrm{~cm}^{-3}$. Other combinations of $\phi$ and $n_{1}$ give deviations of a few hours only.

To conclude, the largest deviations that could be expected in the real-time forecasting in this particular event (since from the coronal hole data we would know about the presence of the high speed stream) are $\Delta T T \approx+3.5 \mathrm{~h}$ and $\Delta T T \approx-10 \mathrm{~h}$, corresponding to $\Delta v_{1} \approx+240 \mathrm{~km} \mathrm{~s}^{-1}$ and $\Delta v_{1} \approx-260 \mathrm{~km} \mathrm{~s}^{-1}$, respectively.

\subsection{Comparison with the numerical model ENLIL}

Since our analytical drag-based model can describe only the propagation of the ICME body and thus cannot reproduce the propagation of the ICME-driven shock, it is instructive to combine our results with the outcome of the numerical heliospheric MHD code ENLIL, which allows the simulation of the solar wind conditions based on the photospheric magnetogram input (http: //ccmc.gsfc.nasa.gov/models/Code_ description.pdf; Odstrcil \& Pizzo 1999; Odstrcil et al. 2004). A version called "ENLIL-with-cone" allows inserting a CME cloud at the inner boundary of ENLIL (21.5 solar radii) and tracing its propagation through the background solar wind. The model runs are available on request at http:// ccmc.gsfc.nasa.gov/, courtesy of the CCMC (Community Coordinated Modelling Center) at the NASA Goddard Space Flight Center.

In the ENLIL-with-cone, the initial-state CME is defined only as a density/velocity/temperature perturbation in the background solar wind, thus lacking the magnetic field specification within the CME body. Consequently, the evolution of the ICME itself is largely artificial, but the propagation of the ICME driven shock is not affected significantly by this drawback. Thus, we will focus primarily to the shock arrival time, meaning that a 

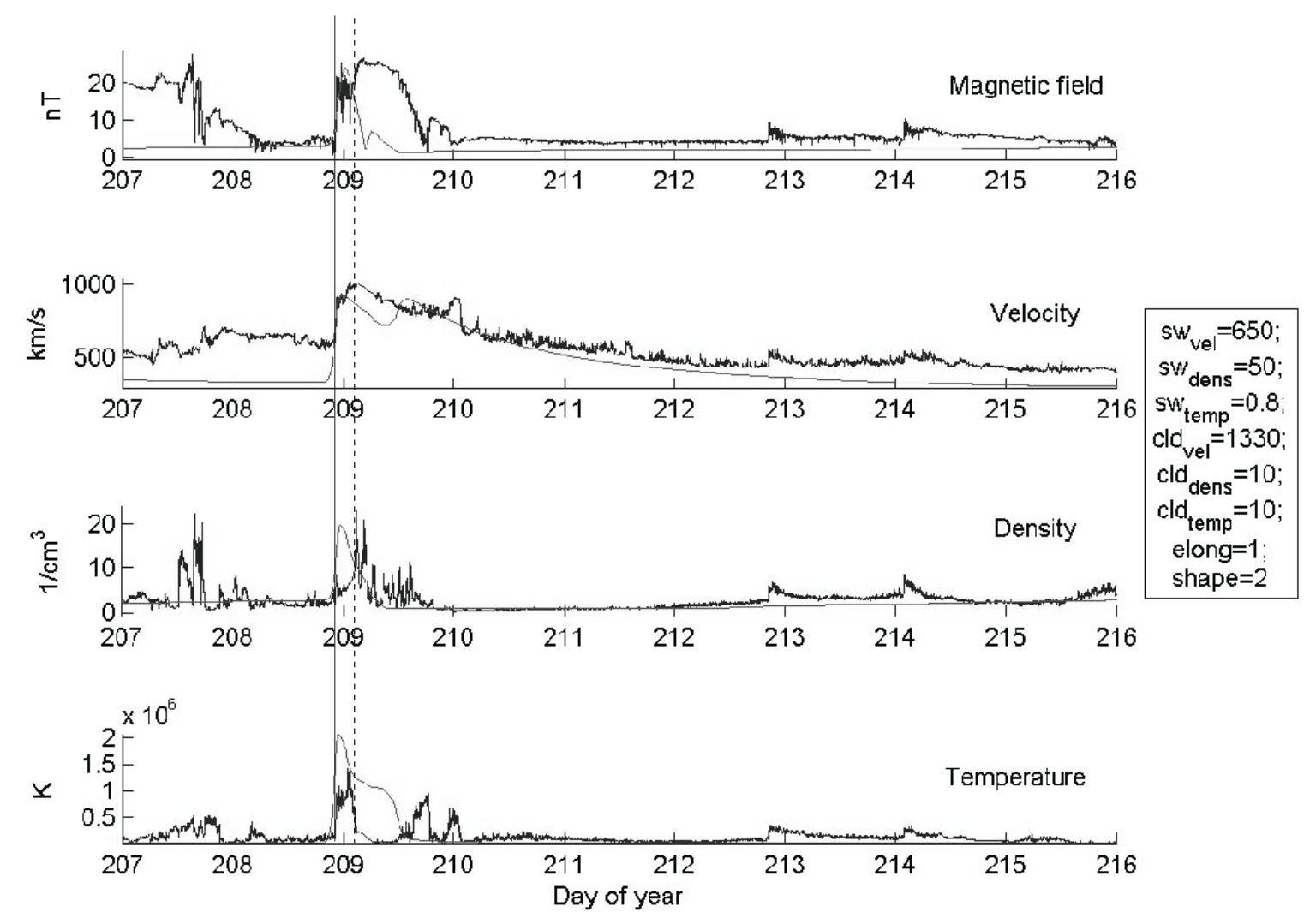

Fig. 6. Comparison of the ENLIL results (smooth thin line) and the ACE in situ measurements (noisy thick line). Note signatures of the solar wind high-speed stream after $D O Y=210$. Vertical full and dashed line indicate the shock and the leading edge of the ICME, respectively.

combination of our model and ENLIL should provide information on both the ICME and the shock.

In Fig. 6 we show the outcome of the ENLIL model run for the following model parameters:

- cloud start date: 2004-07-25;

- cloud start time: 17:49 UT;

- co-latitude: $111^{\circ}\left(0^{\circ}=\right.$ north, $180^{\circ}=$ south $)$;

- longitude: $205^{\circ}\left(90^{\circ}=\right.$ east, $180^{\circ}=$ earth $)$;

- cone radius (i.e., cone half-angle): $75^{\circ}$;

- initial cloud velocity: $v=1330 \mathrm{~km} \mathrm{~s}^{-1}$;

- solar wind input velocity $w=650 \mathrm{~km} \mathrm{~s}^{-1}$;

- solar wind input density $n_{\mathrm{sw}}=50 \mathrm{~cm}^{-3}$;

- solar wind input temperature $T_{\mathrm{sw}}=0.8 \mathrm{MK}$;

- CME density enhancement factor $n_{\mathrm{CME}} / n_{\mathrm{sw}}=10$;

- CME temperature enhancement factor $T_{\mathrm{CME}} / T_{\mathrm{sw}}=10$.

We define the shock arrival-time by the highest gradient in the velocity output of ENLIL. The modeled shock arrival-time is $D O Y=208.91$, which is only about $\approx 1$ h earlier than observed. Given the take-off time $D O Y=207.73$ we find for the shock transit time $T T_{\mathrm{s}}=1.18 \mathrm{~d}=28.4 \mathrm{~h}$. The maximum plasma-flow velocity equals to $942 \mathrm{~km} \mathrm{~s}^{-1}$. Thus, the shock arrival is reproduced very well, as is the flow velocity in the sheath region (according to the ACE data, the shock downstream flow speed was $\left.900 \pm 20 \mathrm{~km} \mathrm{~s}^{-1}\right)$.

\section{Discussion and conclusion}

Our analysis has shown that the ICME transit time and the 1 AU speed depend significantly on all considered parameters: the ICME take-off speed, mass, and width and also the solar wind speed and density. However, there are two limiting cases where the situation simplifies, which is essential for the space weather forecasting.

On one side, there are low-mass and broad ICMEs (i.e., lowdensity ICMEs), whose transit time is determined primarily by the solar wind speed since the ICME adjusts to the solar wind already very close to the sun. The strong dependence of the transit time on the solar wind speed explains the so-called "Brueckner's 80 -h rule" (TT $\approx 80 \mathrm{~h}$ in most events; Brueckner et al. 1998).

On the other hand, in the case of massive and narrow ICMEs, the transit time and the 1 AU speed are determined mostly by the ICME take-off speed. The shortest transit times like $T T \lesssim 1$ day can be achieved only by massive CMEs of a very high initial speed $\left(v_{0}>2000 \mathrm{~km} \mathrm{~s}^{-1}\right)$. Furthermore, such an ICME has to move through high-speed solar wind streams of low density.

From the point of view of the space weather forecasting, it is important that the input parameters required for any ICME propagation model are available in real time. For our model these parameters can be obtained from coronagraphic observations of the ICME take-off. From LASCO data we can estimate the CME mass (Vourlidas et al. 2000) as well as its speed and width (Xie et al. 2004). The EUV observations of coronal changes associated with the eruption can help to also estimate the width too. On the other hand, tracking of equatorial coronal holes in EUV or soft X-ray images provides us with characteristics of high-speed streams in the background solar wind (Vršnak et al. 2007). The solar wind speed and density can be also estimated by applying numerical procedures (e.g. Arge \& Pizzo 2000). Although all of these estimates are of a limited accuracy, they should provide an estimate of the ICME arrival time with an uncertainty of several hours, at least in rather simple situations like that in the event of 25-27 July 2004. 
Finally, we note that one of the major assumptions of the presented model is that the ICME propagates through a uniform solar wind flow, which is far from being realistic. Even in the simplest situation without any equatorial coronal holes, any ICME event will propagate through a bi-modal solar wind, which will result in a significant deformation of the ICME shape. The nose of ICME will move through a slow/dense solar wind, whereas the ICME flanks will travel through a faster and less dense solar wind. As a result of this interaction, the ICME will get a distinct concave shape, where the ICME segments close to the ecliptic plane will lag behind segments at higher heliocentric latitudes (cf. Odstrcil et al. 2004).

In a situation where the slow-wind region is confined to the near-ecliptic latitudes, this effect does not complicate the ICMEarrival prediction, since we know that the Earth will be hit by the ICME segment that travels through the slow solar wind, which can straightforwardly be used as the calculation input. The off-ecliptic segments travel faster, but that does not matter for the forecasting, since we are interested only in the ICME arrival at Earth. However, a similar effect is expected in the presence of equatorial coronal holes, which create streams of fast wind also in the ecliptic plane. Of course this complicates the prediction, since one has to estimate whether the Earth-directed ICME segment is affected by such a stream. In this situation it is inevitable to consult models which describe the background solar wind, and estimate if the Earth-directed ICME segment will travel predominantly through a slow or the fast solar wind stream.

Acknowledgements. The research leading to the results presented in this paper has received funding from European Community's Seventh Framework Programme (FP7/2007-2013) under grant agreement No. 218816. C.M. thanks the Austrian Science Foundation (Fonds zur Förderung der wissenschaftlichen Forschung) for support under project P20145-N16. We are grateful to the LASCO/SoHO, ACE, Wind teams for their open data policy, and to the Community Coordinated Modeling Center at the NASA Goddard Flight Center for carrying out ENLIL runs for the 25 July 2004 event.

\section{References}

Arge, C. N., \& Pizzo, V. J. 2000, J. Geophys. Res., 105, 10465

Borgazzi, A., Lara, A., Echer, E., \& Alves, M. V. 2009, A\&A, 498, 885

Brueckner, G. E., Howard, R. A., Koomen, M. J., et al. 1995, Sol. Phys., 162, 357

Brueckner, G. E., Delaboudiniere, J.-P., Howard, R. A., et al. 1998, Geophys. Res. Lett., 25, 3019

Cantó, J., González, R. F., Raga, A. C., et al. 2005, MNRAS, 357, 572

Cargill, P. J. 2004, Sol. Phys., 221, 135

Cargill, P. J., Chen, J., Spicer, D. S., \& Zalesak, S. T. 1996, J. Geophys. Res., 101,4855

Chen, J. 1989, ApJ, 338, 453

Cho, K.-S., Moon, Y.-J., Dryer, M., et al. 2003, J. Geophys. Res., Space Phys., 108,1445

Dryer, M., Smith, Z., Fry, C. D., et al. 2004, Space Weather, 2, 9001
Feng, X. S., Zhang, Y., Sun, W., et al. 2009, J. Geophys. Res., Space Phys., 114, 1101

Fisher, R. R., \& Munro, R. H. 1984, ApJ, 280, 428

González-Esparza, J. A., Lara, A., Pérez-Tijerina, E., Santillán, A., \& Gopalswamy, N. 2003, J. Geophys. Res., Space Phys., 108, 1039

Gopalswamy, N., Lara, A., Lepping, R. P., et al. 2000, Geophys. Res. Lett., 27, 145

Gopalswamy, N., Lara, A., Yashiro, S., Kaiser, M. L., \& Howard, R. A. 2001, J. Geophys. Res., 106, 29207

Gopalswamy, N., Yashiro, S., Liu, Y., et al. 2005, J. Geophys. Res., Space Phys., 110,9

Gosling, J. T., Bame, S. J., McComas, D. J., \& Phillips, J. L. 1990, Geophys. Res. Lett., 17, 901

Koskinen, H. E. J., \& Huttunen, K. E. J. 2006, Space Sci. Rev., 124, 169

Lara, A., \& Borgazzi, A. I. 2009, in IAU Symp., ed. N. Gopalswamy, \& D. F. Webb, 257, 287

Leblanc, Y., Dulk, G. A., \& Bougeret, J.-L. 1998, Sol. Phys., 183, 165

Manchester, W. B., Gombosi, T. I., Roussev, I., et al. 2004, J. Geophys. Res., Space Phys., 109, 2107

Manoharan, P. K. 2006, Sol. Phys., 235, 345

McKenna-Lawlor, S. M. P., Dryer, M., Kartalev, M. D., et al. 2006, J. Geophys. Res., Space Phys., 111, 11103

Michałek, G., Gopalswamy, N., \& Yashiro, S. 2003, ApJ, 584, 472

Michałek, G., Gopalswamy, N., Lara, A., \& Manoharan, P. K. 2004, A\&A, 423, 729

Odstrcil, D., \& Pizzo, V. J. 1999, J. Geophys. Res., 104, 28225

Odstrcil, D., Riley, P., \& Zhao, X. P. 2004, J. Geophys. Res., Space Phys., 109, 2116

Oler, C. 2004, Space Weather, 2, 8001

Pizzo, V. J., Hill, S. M., Balch, C. C., et al. 2005, Sol. Phys., 226, 283

Schwenn, R., dal Lago, A., Huttunen, E., \& Gonzalez, W. D. 2005, Ann. Geophys., 23, 1033

Smart, D. F., \& Shea, M. A. 1985, J. Geophys. Res., 90, 183

Smith, Z. K., Dryer, M., McKenna-Lawlor, S. M. P., et al. 2009, J. Geophys. Res., Space Phys., 114, 5106

Taktakishvili, A., Kuznetsova, M., MacNeice, P., et al. 2009, Space Weather, 7, 3004

Tappin, S. J. 2006, Sol. Phys., 233, 233

Vourlidas, A., Subramanian, P., Dere, K. P., \& Howard, R. A. 2000, ApJ, 534, 456

Vršnak, B. 2001a, Sol. Phys., 202, 173

Vršnak, B. 2001b, J. Geophys. Res., 106, 25249

Vršnak, B., \& Gopalswamy, N. 2002, J. Geophys. Res., Space Phys., 107, 1019 Vršnak, B., \& Žic, T. 2007, A\&A, 472, 937

Vršnak, B., Ruždjak, D., Sudar, D., \& Gopalswamy, N. 2004, A\&A, 423, 717

Vršnak, B., Temmer, M., \& Veronig, A. M. 2007, Sol. Phys., 240, 315

Vršnak, B., Vrbanec, D., \& Čalogović, J. 2008, A\&A, 490, 811

Xie, H., Ofman, L., \& Lawrence, G. 2004, J. Geophys. Res., Space Phys., 109, 3109

Xue, X. H., Wang, C. B., \& Dou, X. K. 2005, J. Geophys. Res., Space Phys., 110,8103

Yashiro, S., Gopalswamy, N., Michalek, G., et al. 2004, J. Geophys. Res., Space Phys., 109, 7105

Yousef, S., El Nawawy, M. S., El-Nazer, M., \& Yousef, M. 2005a, in Coronal and Stellar Mass Ejections, ed. K. Dere, J. Wang, \& Y. Yan, IAU Symp., 226, 477

Yousef, S., El-Nazer, M. M., \& Bebars, A. 2005b, in Coronal and Stellar Mass Ejections, ed. K. Dere, J. Wang, \& Y. Yan, IAU Symp., 226, 145

Zhang, J., Dere, K. P., Howard, R. A., \& Bothmer, V. 2003, ApJ, 582, 520

Zhao, X. P., Plunkett, S. P., \& Liu, W. 2002, J. Geophys. Res., Space Phys., 107, 1223 